Fabricating Optical Fiber Imaging Sensors Using Inkjet Printing Technology: a pH Sensor Proof-of-Concept

J. C. Carter, R. M. Alvis, S. B. Brown, K. C. Langry, T. S. Wilson, M. T. McBride, M. L. Myrick, W. R. Cox, M. E. Grove, B. W. Colston

March 2, 2005

Biosensors and Bioelectronics 
This document was prepared as an account of work sponsored by an agency of the United States Government. Neither the United States Government nor the University of California nor any of their employees, makes any warranty, express or implied, or assumes any legal liability or responsibility for the accuracy, completeness, or usefulness of any information, apparatus, product, or process disclosed, or represents that its use would not infringe privately owned rights. Reference herein to any specific commercial product, process, or service by trade name, trademark, manufacturer, or otherwise, does not necessarily constitute or imply its endorsement, recommendation, or favoring by the United States Government or the University of California. The views and opinions of authors expressed herein do not necessarily state or reflect those of the United States Government or the University of California, and shall not be used for advertising or product endorsement purposes. 


\section{Fabricating Optical Fiber Imaging Sensors Using Inkjet Printing Technology: a pH Sensor Proof-of-Concept}

\section{UCRL-JRNL-210157}

J. Chance Carter ${ }^{\text {a*}}$, Rosa M. Alvis ${ }^{\text {a }}$, Steve B. Brown ${ }^{\mathrm{a}}$, Kevin C. Langry ${ }^{\mathrm{a}}$, Thomas S. Wilson $^{\mathrm{a}}$, Mary T. McBride ${ }^{\mathrm{a}}$, M. L. Myrick ${ }^{\mathrm{b}}$, W. Royall Cox ${ }^{\mathrm{c}}$, Michael E. Grove ${ }^{\mathrm{c}}$, and Bill W. Colston ${ }^{\mathrm{a}}$

${ }^{a}$ Lawrence Livermore National Laboratory, 7000 East Ave, Livermore, CA 94550

${ }^{\mathrm{b}}$ University of South Carolina, Dept. of Chemistry and Biochemistry, Columbia, SC 29208.

${ }^{c}$ Microfab Technologies, Inc., Plano Texas, 75074 
*Corresponding author. Tel.: 925-424-4005; fax: 925-423-9014

E-mail address: Carter45@1lnl.gov (J.C. Carter).

Abstract

We demonstrate the feasibility of using Drop-on-Demand microjet printing technology for fabricating imaging sensors by reproducibly printing an array of photopolymerizable sensing elements, containing a $\mathrm{pH}$ sensitive indicator, on the surface of an optical fiber image guide. The reproducibility of the microjet printing process is excellent for microdot (i.e. micron-sized polymer) sensor diameter (92.2 \pm 2.2 microns), height $(35.0 \pm 1.0$ microns), and roundness $(0.00072 \pm 0.00023) . \mathrm{pH}$ sensors were evaluated in terms of $\mathrm{pH}$ sensing ability ( $\leq 2 \%$ sensor variation), response time, and hysteresis using a custom fluorescence imaging system. In addition, the microjet technique has distinct advantages over other fabrication methods, which are discussed in detail. 
Keywords: optical fiber, image guide, $\mathrm{pH}$ sensor, imaging, biosensor, microjet, inkjet, microdot

\section{Introduction}

The most promising approach to fiber-based $\mathrm{pH}$ sensing has been the confinement [e.g. covalent (Peterson et al., 1980; Saari and Seitz, 1982; Gehrich et al., 1986; Munkholm et al., 1986; Jordan and Walt, 1987; Nivens et al., 1998), electrostatic (Zhujun and Seitz, 1984; Nivens et al., 2002)] of pH sensitive indicators in substrates attached to the fiber surface. Traditional methods for fabricating fiber-based $\mathrm{pH}$ sensors involve attachment of the substrate through mechanical (Peterson et al., 1980; Saari and Seitz, 1982; Gehrich, et al., 1986; Zhujun and Seitz, 1984), dip coating (Nivens et al., 1998, 2002), or photopolymerization methods (Munkholm et al., 1986; Jordan and Walt, 1987). Mechanical methods vary but most designs utilize tubing (e.g. capillary) filled with indicating reagent. In some cases, the substrate is directly bound (Fuh et al., 1987) (e.g. epoxy) to the fiber surface. Sensors of this type are typically fabricated in two principal steps. The steps involve immobilizing the indicator chemistry on a solid support material, and subsequently attaching this to the fiber. This method gives better reproducibility and is widely used (Wolfbeis, 1991). However, a survey of the literature shows that sensors fabricating in this manner are limited to single analyte measurements. Dip coating methods are commonly used in many sol-gel sensor preparations and typically produce micron-thick sensing membranes per dip, with the resulting membrane(s) covering the entire surface of the fiber. Unlike mechanical methods, the sensing layer can be produced in one step since the fiber tip is dipped in a formulation containing both the indicator chemistry and the solid support chemistry. Multiple coatings of different indicating chemistries can be sequentially added to the same fiber, producing multianalyte sensors (Nivens et al., 2002; Schiza et al., 2001). Such multianalyte sensor designs can suffer from issues of chemical compatibility and cross sensitivity. Sensors fabricated by dip coating have not been shown to be reproducible and do not offer spatial discrimination of the individual sensing layers, since each target analyte must interact with the indicator chemistry of a particular layer and produce an optically distinct signal (e.g. fluorescence or absorption).

Photopolymerization methods are among the earliest methods used for fiber-based sensor fabrication. In recent years, Walt et al advanced this method by demonstrating that unique patterns of indicator chemistries could be covalently attached directly to the tips of optical fiber bundles, comprised of thousands of densely packed fibers (Healey et al., 1995; Healey and Walt, 1997; Barnard and Walt, 1991). Specifically, these polymerized arrays of indicator chemistries were produced by immersing the optical fiber tip in a polymerizable indicator chemistry and selectively "growing" the indicator chemistries on the end of the optical fiber strands via ultraviolet UV) radiation photopolymerization. These sensor arrays were then spatially discriminated using simple imaging techniques. Multianalyte sensors can be fabricated by immersing the fiber tip sequentially in different polymerizable solutions followed by photopolymerization. However, the order in which the sensing elements were added to the fiber surface was critical because of cross sensitivity issues (Ferguson et al., 1997). Furthermore, these arrays are non-uniform, resulting from the lack of control during the photopolymerization step. This leads to sensors that are not reproducible in their response. 
Building upon Walt's work, we demonstrate the feasibility of using Drop-onDemand microjet printing technology (Wallace et al., 2002) for fabricating imaging sensors by printing an array of photopolymerizable sensing elements on the surface of an optical fiber image guide. The microjet printing process produces highly reproducible droplets via a piezoelectric driven orifice, resulting in a very uniform sensor array. For these initial studies, a $\mathrm{pH}$ sensitive indicator was immobilized in a polymeric support and tested using a fluorescence imaging apparatus. Several key optical and physical properties of the microdot sensor array are evaluated in terms of morphology, response time, and $\mathrm{pH}$ sensing reproducibility.

\section{Experimental}

The printed indicator chemistry was comprised of a proprietary optical polymer (i.e. epoxy) that was photopolymerized from a mixture of glycidyl ethers of dihydroxy compounds including cyclohexanedimethanol diglycidyl ether, glycerol diglycidyl ether, butanediol diglycidyl ether, and ethylene glycol diglycidyl ether, in which a $\mathrm{pH}$ sensitive dye, fluorescein (Sigma-Fluka), was dissolved at $0.6 \% \mathrm{w} / \mathrm{w}$. The concentration of fluorophore in the polymer was based on prior experience of one author. Reactive materials were purchased from Aldrich Chemical Co. and Monomer-Polymer Laboratories, and used as received. All photopolymerization was performed using the Green $\operatorname{Spot}^{\mathrm{TM}}$ (300-480nm) from UV Sources, Inc. The Lewis acid initiator species $\mathrm{SbF}_{6}$ was created in situ by UV photolysis $(2000 \mathrm{~mW})$ from a commercial triaryl compound present at a $0.5 \% \mathrm{w} / \mathrm{w}$ level. Buffered solutions were prepared using Hydrion buffers.

Optical image guides (Sumitoma, IGN-05/06) were polished (1 micron finish) and cleaned with acetone. After evaporation of solvent, image guides were dipped in a commercially available fluoroaliphatic copolymer solution (3M Inc., FC-724 proprietary formulation), which also contains a low concentration of alkyltrialkyloxysilane coupling agent. The image guides were then baked for $\sim 45 \mathrm{~min}$ at $140^{\circ}$, resulting is a single microns-thick, optically transparent coating on the fiber surface with a surface energy expected to be $\sim 15$ dynes $/ \mathrm{cm}$ as reported in the $3 \mathrm{M}$ literature. Adhesion of the coating to the optical fiber is presumed to be a combination of mechanical adhesion, van der Waals attraction, and some covalent bonding through condensation of surface silanol groups on the optical fiber with the alkyloxysilane groups of the coating during the baking process. Adhesion of the subsequently polymerized microdot sensors (epoxy chemistry) to the fluoropolymer coating is presumed to be a combination of mechanical interlocking and van der Waal's forces. The fluoroaliphatic copolymer likely contains polyfluoroacrylate, which would allow hydrogen bonding between carbonyl groups and the hydroxyl groups of the epoxy. Although rigorous adhesion characterization was not performed, the microdots remained intact on the fiber surface after repeated cycles of exposure to aqueous environments with subsequent drying.

The custom fluorescence imaging system, a similar system previously described (Carter et al., 1999), utilizes a liquid nitrogen-cooled, charge-couple device (Princeton Instruments, Model LN/CCD-512TKB/1) photodetector and a high intensity blue lightemitting-diode (LED) for excitation. The imaging system was designed to magnify the distal portion of the optical image guide 10 fold. The resulting image of the fiber tip covered 203 pixels of the CCD (no pixel binning). For all fluorescence measurements, $7 \times 7$ on-chip pixel binning was used. This resulted in a $29 \times 29$ grid of superpixels in 
which there was no overlap in signal from adjacent microdot sensing elements. The images used to calculate the calibration curve shown in Figure 3 were acquired with 10 coadditions, $25 \mathrm{sec}$ exposures each. Images used to calculate the response time data shown in Figure 4 were acquired with $12 \mathrm{sec}$ exposures.

$\mathrm{pH}$ measurements below $\mathrm{pH} 6$ were hindered by matrix effects. The relative error for the $\mathrm{pH} 6$ and $\mathrm{pH} 3-5$ (not shown) measurements was greater by a factor of 3-4 compared to the relative error for all data points from $\mathrm{pH} 6.8$ to 9 . The pooled relative error for data between $\mathrm{pH} 6.8$ and 9 was $1.3 \%$. When the data for $\mathrm{pH} 6$ was included in the calculation, the pooled relative error was $2.2 \%$. The $2 \%$ variation we report in the text is actually on the high end of the measurement.

\section{Results and Discussion}

The process for inkjet printing indicator chemistries on an optical substrate is similar to that used to produce micro-optical components (Cox et al., 1995; Cox et al., 1996; Chen et al., 2002). This technology, known as Drop-on-Demand microjet printing, is a process that utilizes a piezo driven orifice, which emits a highly reproducible droplet (i.e. indicator chemistry) each time an appropriate driving pulse produces a displacement of the piezoelectric element surrounding the orifice. Figure 1a shows an image of droplet emission from the piezo driven orifice. With the aid of stroboscopic illumination, this image is actually the superposition of $\sim 1000$ individual droplets. The clarity of the monomer droplet in the lower part of Figure 1a indicates the time and spatial precision of the microjet process. Other key features of the microjet system (Fig 1b) include motion control actuators for accurate positioning of the substrate (i.e. optical fiber) during printing, a UV light tube for photopolymerizing printed monomers, and a cleanair/exhaust system for preventing airborne contamination.

The microjet system was used to print microdot arrays of indicator chemistries on the tips of optical fiber image guides. The capabilities of the microjet process for printing chemistries on an optical image guide are shown in the darkfield images of Figure 2a-d. The top-view image of Figure 2a shows 7 microdots 'printed' in a 6-around-1 configuration on the polished surface of a 500 micron optical image guide. The diameter of the circle through the centers of the circumferentially printed microdots is 260 microns, indicating this pattern easily fits on the end of the image guide. The reproducibility of the microjet printing process is excellent for microdot sensor diameter (92.2 \pm 2.2 microns), height ( $35.0 \pm 1.0$ microns), and roundness $(0.00072 \pm 0.00023)$, which is calculated by dividing the difference between maximum and minimum diameters by the average diameter. Figure $2 \mathrm{~b}$ shows a side view of the seven microdots and provides visual detail about the array aspect ratio (i.e. diameter vs. height). This ratio is controllable by adjusting physical characteristics (e.g. surface tension, viscosity) of the monomer formulation using surface active materials, as example Fluoroad FC171 (3M, Inc.), and temperature $\left(75^{\circ} \mathrm{C}\right)$ of the fluid in the printhead, by adjusting the surface energy of the optical fiber using surface modifiers (e.g. fluoroaliphatic copolymer), and by adjusting the number and diameter (orifice size) of deposited droplets. In all experiments, each microdot is a single droplet expelled from a 40-micron orifice, and all image guide fibers are treated prior to printing in this study with a fluoroaliphatic copolymer surface modifier. Figures $2 \mathrm{c}$ and $\mathrm{d}$ are the expanded top view and side view, respectively, of a single microdot and clearly show the microdot symmetrical 
morphology. It should be noted that beneath the transparent microdot of Figure $2 \mathrm{c}$, the individual pixels comprising the optical image guide are clearly discernable due to a lensing effect caused by the microdot morphology. In addition to morphological characteristics, the fluorescence intensity of 30 fluorescein-doped microdots were measured and compared using an imaging spectrometer and determined to vary by 2 percent or less.

Although this example of an optical sensor system uses only one indicator, it demonstrates the unique capability of inkjet technology to place multiple microdots, each possibly containing a different fluorophore, on the end of the optical imaging fiber. Any indicator compound that will form a solid solution in a polyether or polyacrylate produced by photopolymerization is a suitable candidate.

\subsection{Sensor calibration/variability}

The $\mathrm{pH}$ sensing ability of the fluorescein-doped microdots was evaluated using a custom fluorescence imaging system. Sensor variability of the microdot array was minimal across the range of $\mathrm{pH} 6$ to 9 as shown in Figure 3. In the $\mathrm{pH}$ range 6.8 to 8 , the response is linear. Above $\mathrm{pH} 8$, the change in fluorescence intensity with increasing $\mathrm{pH}$ is less due to the $\mathrm{pKa}$ of the immobilized fluorescein indicator. Though fluorescein is typically used over a wider $\mathrm{pH}$ range than shown, we were unable to detect significant changes in $\mathrm{pH}$ below $\mathrm{pH}$ 6. We attribute this to an undesirable residual or buffering effect within the microdots caused by the use of the Lewis acid catalyst species, $\mathrm{SbF}_{6}{ }^{-}$ion and UV radiation for polymerizing the fluorescein-doped formulation. It is important to note that because of uneven illumination (i.e. excitation) of the individual fluorescein immobilized microdot sensors, all data for each microdot was normalized to the fluorescence intensity at $\mathrm{pH} 9$ and plotted as the relative change in fluorescence intensity versus $\mathrm{pH}$.

\subsection{Sensor response time/hysteresis}

The time response of the 6-around-1 microdot array to changes in $\mathrm{pH}$ was evaluated. For these experiments, the fiber tip was alternated between $\mathrm{pH} 9$ and $\mathrm{pH} 6.8$ buffered solutions while acquiring successive images. In Figure 4, the response curves of 7 microdots over 3 cycles of $\mathrm{pH}$ changes between $\mathrm{pH} 9$ to $\mathrm{pH} 6.8$ were identical and show a $t_{100}$ (time to $100 \%$ of total response) response time of $\sim 25 \mathrm{sec}$ for decreasing or increasing changes. These plots were also normalized due to uneven illumination of the microdot array as previously discussed. The large spike that is evident at the edge of the transition from $\mathrm{pH} 9$ to 6.8 (cycle 3) was intentional to show how these sensors respond to brief exposure to air while changing between $\mathrm{pH}$ buffered solutions. The lack of hysteresis in the response curves indicates no photobleaching and/or dye leaching.

\section{Conclusions}

Microjet printing technology is a viable tool for fabricating fiber-based imaging sensors. Although only $\mathrm{pH}$ sensing was demonstrated in this pilot study, the microjet technique is potentially amenable to fabricating multianalyte sensors to simultaneously measure other biologically important parameters such as blood/gas and other ions. Sensors fabricated in this manner do not have issues of cross sensitivity or chemical compatibility since each indicator chemistry can be attached to the fiber surface 
independently (i.e. microdots are never exposed to other chemistries). This is in contrast to dip coating and other photopolymerization techniques. Multianalyte sensors, in which different indicators would be used, could greatly benefit from this technique. Another important feature of this technique is the excellent uniformity of the polymer sensor arrays on the fiber surface. Such uniformity has not been demonstrated with any of the other techniques discussed in this paper. The ability to fabricate reproducible sensors could enable large-scale production of fiber sensors without the need to calibrate each individual sensor.

\section{Acknowledgments}

This work was performed under the auspices of the U.S. Department of Energy by the University of California, Lawrence Livermore National Laboratory under contract No. W-7405-Eng-48. 


\section{References}

- Barnard, S.M., Walt, D.R., 1991. A fiberoptic chemical sensor with discrete sensing sites. Nature 353 (6342) 338-340.

- Carter, J.C., Egan, W.J., Nair, R.B., Murphy, C.J., Morgan, S.L., Angel, S.M., 1999. Fiber-optic imaging for in-situ chemical measurements. SPIE Proceed. 3540, 210 221.

- Chen, T., Cox, W.R., Lenhard, D., Hayes, D.J., 2002. Microjet printing of high precision microlens array for packaging of fiber-optic components. SPIE Proc. 4652, 136-141.

- Cox, W.R., Chen, T., Ussery, D., Hayes, D.J., Tatum, J.A., MacFarlane, D.L., 1996. Microjetted lenslet-tipped fibers. Optics Communication 123(4-6), 492-496.

- Cox, W.R., Hayes, D.J., Chen, T., Ussery, D.W., 1995. Fabrication of micro-optics by microjet printing. SPIE Proceed. 2383, 110-115.

- Ferguson, J.A., Healey, B.G., Bronk, K.S., Barnard, S.M., Walt, D.R., 1997. Simultaneous monitoring of $\mathrm{pH}, \mathrm{CO}_{2}$ and $\mathrm{O}_{2}$ using an optical imaging fiber. Anal. Chim. Acta 340, 123-131.

- Fuh, M.R.S., Burgess, L.W., Hirschfeld, T., Christian, G.D., Wang, F., 1987. Single fibre optic fluorescence $\mathrm{pH}$ probe. Analyst 112 (8), $1159-1163$.

- Gehrich, J.L., Lubbers, D.W., Opitz, N., Hansmann, D.R., Miller, W.W., Tusa, J.K., Yafuso, M., 1986. Optical fluorescence and its application to an intravascular bloodgas monitoring system. IEEE Trans. Biomed. Eng. 33, 117-132.

- Healey, B.G., Foran, S.E., Walt, D.R., 1995. Photodeposition of micrometer-scale polymer patterns on optical imaging fibers. Science 269 (5227), 1078-1080.

- Healey, B.G., Walt, D.R., 1997. Fast temporal response fiber optic chemical sensors based on the photodeposition of micrometer-scale polymer arrays. Anal. Chem. 69, 2213-2216.

- Jordan, D.M., Walt, D.R., 1987. Physiological pH fiber optic chemical sensor based on energy-transfer. Anal. Chem. 59, 437-439.

- Munkholm, C., Walt, D.R., Milanovich, F.P., Klainer, S.M., 1986. Polymer modification of fiber optic chemical sensors as a method of enhancing fluorescence signal for $\mathrm{pH}$ measurements. Anal. Chem. 58, 1427-1430.

- Nivens, D.A., Schiza, M.V., Angel, S.M., 2002. Multilayer sol-gel membranes for optical sensing applications: single layer $\mathrm{pH}$ and dual layer $\mathrm{CO}_{2}$ and $\mathrm{NH}_{3}$ sensors. Talanta 58(3), 543-550.

- Nivens, D.A., Zhang, Y., Angel, S.M., 1998. A fiber optic pH sensor using basecatalyzed organo-silica sol-gel. Anal. Chim. Acta 376, 235-245.

- $\quad$ Peterson, J.I., Goldstein, S.R., Fitzgerald, R.V., Buckhold, D.K., 1980. Fiber optic pH probe for physiological use. Anal. Chem. 52, 864-869.

- Saari, L.A., Seitz, W.R., 1982. pH sensor based on immobilized fluoresceinamine. Anal. Chem. 54, 821-823. 
- $\quad$ Schiza, M.V., Nelson, M.P., Myrick, M.L., Angel, S.M., 2001. Use of a 2D to 1D dimension reduction fiber-optic array for multiwavelength imaging sensors. Appl. Spectrosc. 55(2), 217-226.

- Wallace, D.B., Cox, W.R., Hayes, D.J., 2002. In: Pique, A., Chrisey, D.B., (Eds.), Direct Write Using Ink-Jet Techniques, Academic Press, New York, Chapter 7.

- Wolfbeis, O. S., (Ed.), 1991. Fiber-Optic Chemical Sensors and Biosensors Volume 1, CRC Press, Boca Raton, FL, pp. 77.

- Zhujun, Z., Seitz, W.R., 1984. A fluorescence sensor for quantifying $\mathrm{pH}$ in the range from 6.5 to 8.5. Anal. Chim. Acta 160, 47-55. 
Figure 1: (a) A stroboscopically illuminated image of 50 micron diameter droplets ( 1000 superimposed) of polymer-based indicator chemistry emitted from the piezodriven microjet orifice, and a (b) schematic of the Drop-on-Demand microjet system used in printing patterned arrays of indicator chemistries on optical image guides.

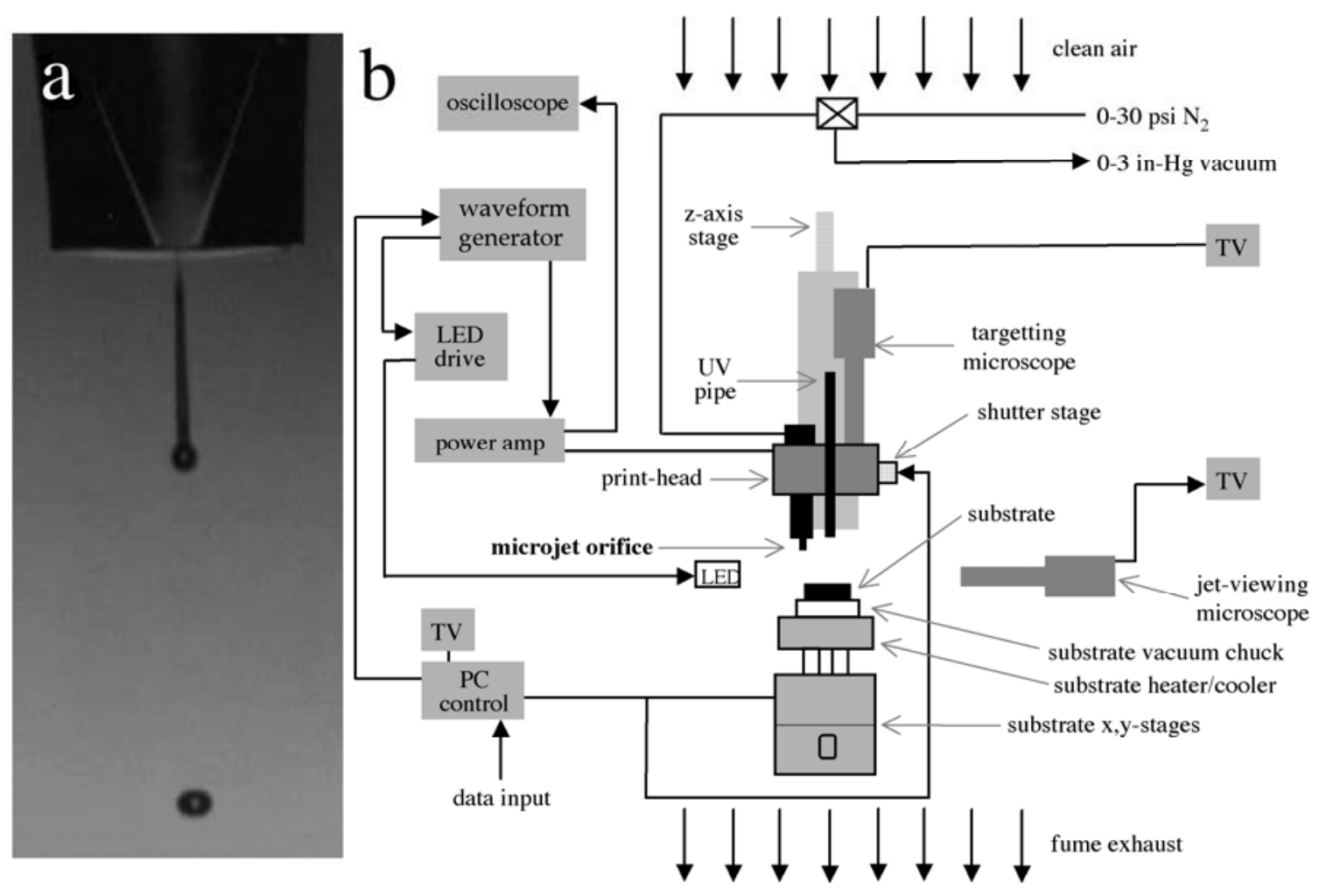


Figure 2: (a) Top view and (b) side view of seven 92 micron diameter microdots printed on a 500 micron diameter optical image guide and the (c) expanded top view and (d) side view of a single 92 micron diameter microdot.
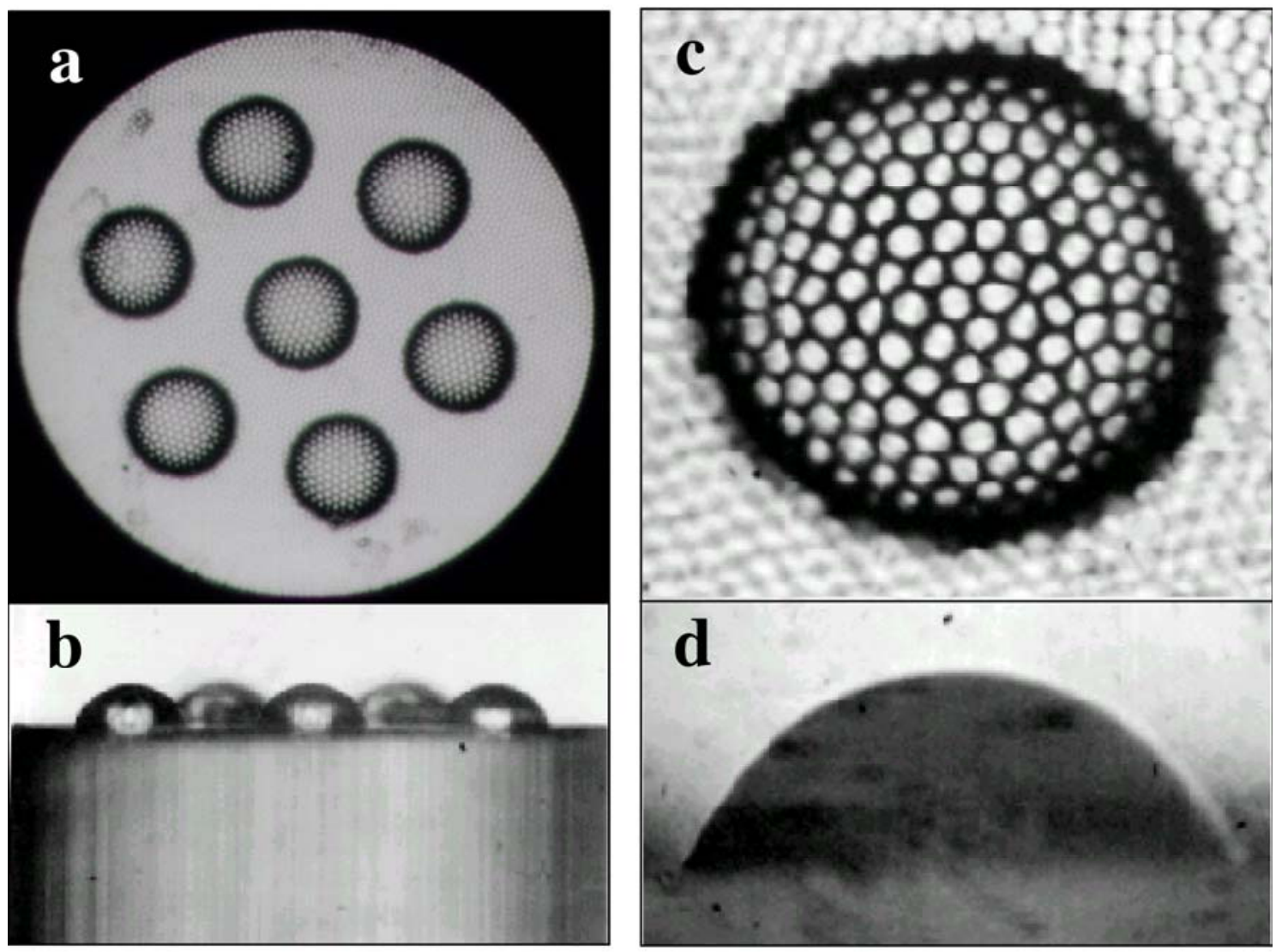
Figure 3: Plot of normalized fluorescence intensity vs. $\mathrm{pH}$ for a 6-around-1 microdot array. Error bars show \pm 1 standard deviation.

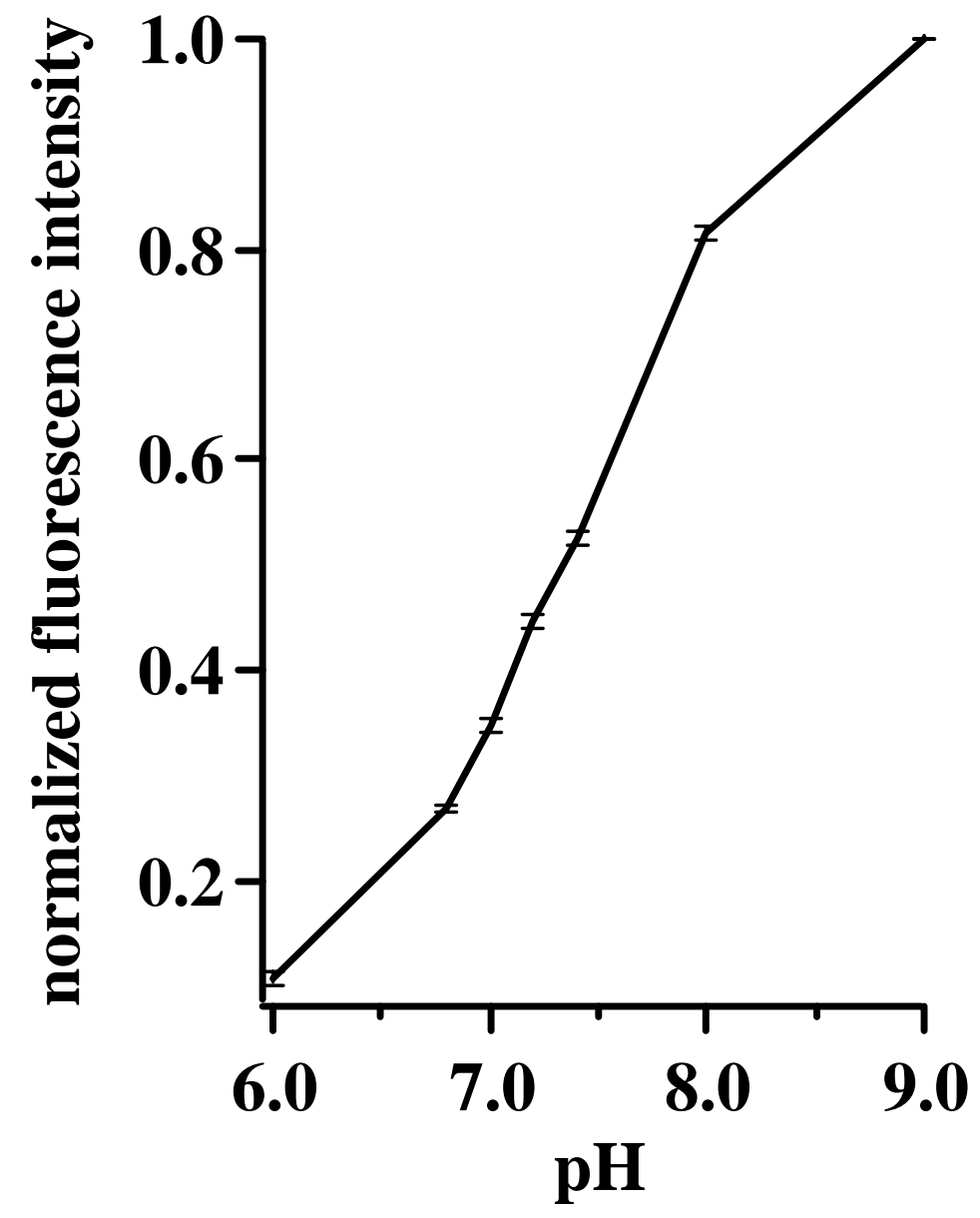


Figure 4: Relative $\mathrm{pH}$ response time curves of a 6-around-1 microdot array between $\mathrm{pH}$ 9 and 6.8 indicating a $t_{100}$ response time of $\sim 25$ seconds.

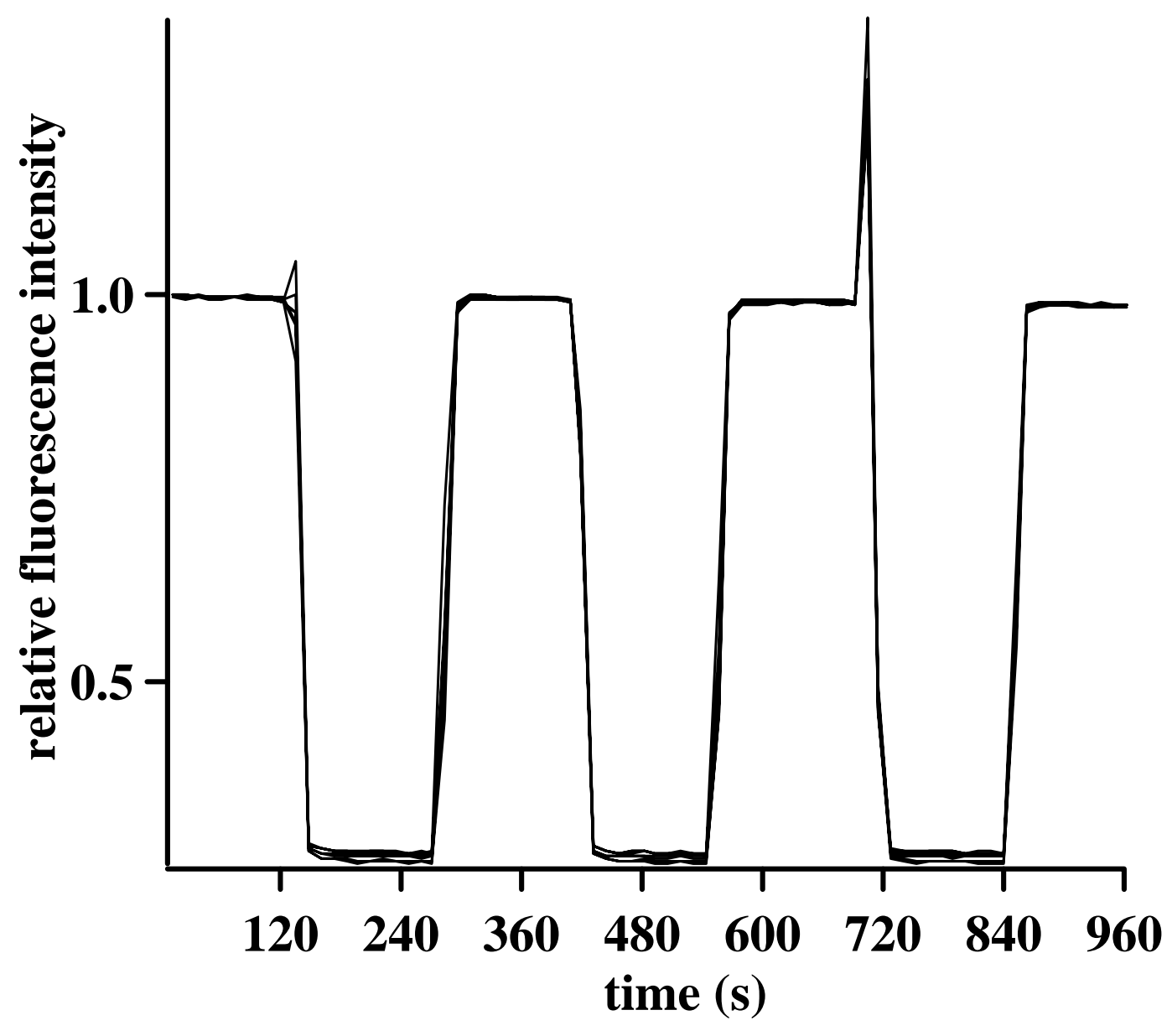


